\title{
The contribution of olfaction to taste discrimination
}

\author{
ARNOLD HYMAN, THOMAS MENTZER, and LEO CALDERONE \\ University of New Haven, West Haven, Connecticut 06516
}

\begin{abstract}
To assess the contribution that odor makes to tasting, subjects made forced-choice discriminations of orange juice, vinegar, and chocolate syrup using taste and smell combined, taste alone, or smell alone. The taste-alone group performed more poorly than the taste-smell group on two of the three substances at low concentrations on the detection task. On vinegar the smell-alone group performed close to chance levels and the taste-only group was comparable to the taste-smell group. On the recognition task, all three groups discriminated poorly compared to detection performance except for the taste-smell group on chocolate syrup. It was concluded that the contribution of odor to tasting is related to the intensity of the odor.
\end{abstract}

The interrelationship of the senses of taste and smell has been a topic of some interest ever since BrillatSavarin's early observation $(1825 / 1971$, p. 39$)$ that the flavor of food is largely influenced by its aroma. It is also likely that tactile and temperature sensations contribute significantly to the tasting experience (Rubin, 1936).

The taste-smell interaction has most often been studied by observation of subjects who have become anosmic through injury to the brain or olfactory nerves. These studies have produced mixed findings, with some authors (Gilliland, 1921; Schneider, 1967) concluding that anosmia produces sizable gustatory deficits and others (Clark, 1968; Clark \& Dodge, 1955a, 1955b; Crosland, Goodman, \& Hockett, 1926) finding anosmics to be nearly normal in taste discrimination.

Mozell, Smith, Smith, Sullivan, and Swender (1969) criticized the use of anosmics in the study of the tastesmell interaction and developed a method that uses normal subjects. They obtained taste identifications of 31 common food substances with and without the use of olfaction and concluded that there were significant deficits when olfaction was eliminated. They did not study performance using smell alone. Although stimulus concentrations were not reported, they apparently were well above threshold, with solids blended and diluted so that viscosities were uniform. Mozell et al. encountered one problem that is common when high concentrations are used. Some substances (e.g., onion, garlic) can be detected at high concentrations because they produce a tactile irritation that has been termed the "trigeminal effect" (Doty, 1975).

The purpose of this experiment was to compare the ability of normal subjects to detect and identify familiar substances using taste and smell combined, taste alone,

The authors wish to thank Dennis Courtney for his contribution to this project. The research was supported by a grant from the University of New Haven. and smell alone. The smell-alone condition was included since it seemed likely that the extent to which the odor of a substance contributes to its flavor is dependent on the intensity of the odor. The stimuli were presented at low concentrations so that the trigeminal effect could be avoided and psychophysical functions could be obtained.

\section{METHOD}

\section{Subjects}

The subjects were 64 males and 26 females, mostly college students, between the ages of 9 and 31 years. Only one subject was less than 17 years of age and six were more than 25 years of age. The subjects were prescreened for respiratory illnesses and chronic olfactory and gustatory disorders. Smokers were used as subjects only if they had not smoked for $3 \mathrm{~h}$ prior to testing.

\section{Procedure}

A pilot study was performed to select taste substances that were $100 \%$ identifiable at maximum concentration. Four substances proved to be $100 \%$ identifiable: orange juice, vinegar, chocolate syrup, and peanut butter. The substances selected and used throughout the experiment were orange juice (Tropicana), white vinegar (Heinz), and chocolate syrup (Hershey). Peanut butter was excluded because of the difficulty involved in making a solution. Each substance was diluted to form six concentrations (shown in Table 1) chosen to cover the range from chance discrimination to perfect discrimination based on pilot study data.

The eyes of all subjects were covered with a cloth blindfold

Table 1

Stimulus Concentrations (Grams of Substance Per $100 \mathrm{ml}$ Water)

\begin{tabular}{cccccc}
\hline \multicolumn{2}{c}{ Orange Juice } & \multicolumn{2}{c}{ Vinegar } & \multicolumn{2}{c}{ Chocolate Syrup } \\
\hline $\mathrm{J} 1$ & .10 & V1 & .02 & $\mathrm{C} 1$ & .05 \\
$\mathrm{~J} 2$ & .15 & V2 & .03 & $\mathrm{C} 2$ & .07 \\
$\mathrm{~J} 3$ & .25 & $\mathrm{~V} 3$ & .05 & $\mathrm{C} 3$ & .10 \\
$\mathrm{~J} 4$ & .40 & $\mathrm{~V} 4$ & .0625 & $\mathrm{C} 4$ & .20 \\
$\mathrm{~J} 5$ & .50 & $\mathrm{~V} 5$ & .10 & $\mathrm{C} 5$ & .25 \\
$\mathrm{~J} 6$ & .65 & $\mathrm{~V} 6$ & .125 & $\mathrm{C} 6$ & .375 \\
\hline
\end{tabular}


fitted with foam rubber pads. The subjects were randomly assigned to one of the following conditions: (1) Taste-Smell (TS)-Subjects in this group used both taste and smell in making their judgments. (2) Taste (T)-Each subject in this group wore a swimmer's nose clip during tasting. These clips were found in the pilot study to prevent nasal inspiration. (3) Smell (S)-These subjects were not permitted to taste any of the solutions. All judgments were based on smell alone.

The following instructions were read to TS subjects. The instructions were modified appropriately for subjects in the $\mathrm{T}$ and $S$ groups.

"We are conducting an experiment in order to determine a person's ability to identify very dilute amounts of common foods. You will be blindfolded and you will be asked to smell and taste three different substances: chocolate, orange juice, and vinegar. You will be given a small cup of water and a small cup of a dilution of one of the three substances, but you will not be told which is water or what substance is being given. You will smell, then taste each cup, then indicate which is the substance and which substance it is. In other words, you must make two judgments. First, you must tell me whether the first or second cup contains the substance, and second, you must identify which of the three substances was presented to you. For each trial, one cup will always contain water and one cup will contain either chocolate, orange juice, or vinegar. Sometimes the water will be presented first, sometimes the substance will be first. Some of the judgments may be quite difficult. Simply make the best judgments you can.

"You must not swallow any of the liquids presented to you. After you smell and taste the first liquid, spit it out into the large cup here. Then take the second liquid, smell and taste it, and spit it out. After you have made your judgments, take a sip of water from the glass, rinse your mouth, and spit it out. Then wait for the next pair of liquids and repeat the procedure. Do not remove the blindfold until you are requested to do so at the end of the experiment.

"Do you understand the procedure? Do you have any questions? Here is a sample of the strongest solution of each of the three substances."

The subject was then given a sample of the strongest solution of each substance. Following this, the subject was tested for 18 trials, receiving each of the six concentrations of the three substances once in random order.

Tasting was conducted using the forced-choice variation of the method of constant stimuli (Kling \& Riggs, 1971, p. 19). On each trial the subject was presented two small plastic cups in succession. One cup contained approximately $15 \mathrm{ml}$ of distilled water and the other contained the same amount of one of the 18 stimulus solutions. The subjects were never told which of the 18 stimuli was being presented, nor did they know whether the stimulus was in the first or second cup. On each trial the subject judged whether the first or second cup contained the taste substance (detection) and then identified the substance by name (orange juice, chocolate, or vinegar), guessing if necessary (recognition). No time restrictions were placed on sampling or judgments. After the judgments were made, the subject rinsed with distilled water. The experimenters waited at least $30 \mathrm{sec}$ between rinsing and the next stimulus presentation.

Four different experimenters participated, testing 10,13, 28 , and 39 subjects, respectively. The subjects were tested individually in a well ventilated room. All substances were presented at room temperature, and each cup was stirred just prior to sampling. The subjects received no feedback of any kind regarding stimulus conditions or judgments. Each testing session lasted approximately $30 \mathrm{~min}$.

\section{RESULTS AND DISCUSSION}

Figure 1 contains the psychophysical functions for the three testing conditions separated according to
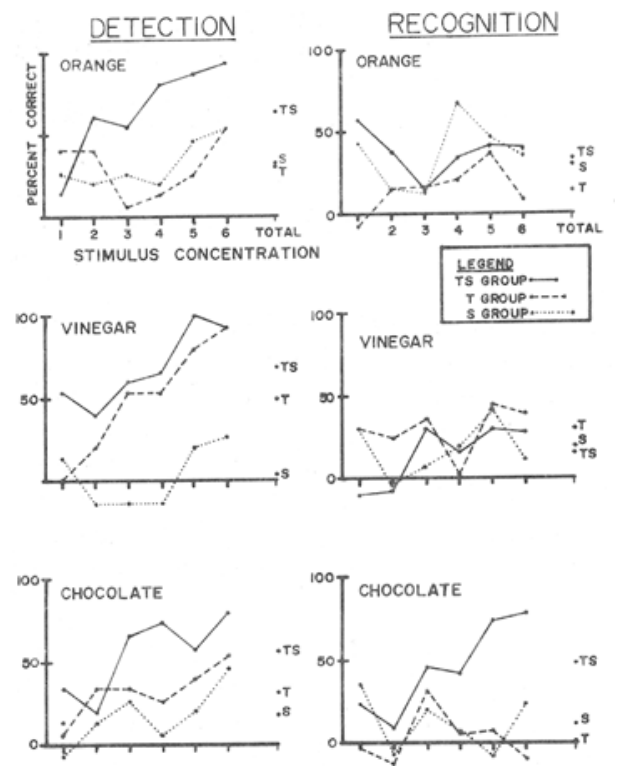

Figure 1. Psychophysical functions for detection (left) and recognition (right) separated by groups and taste substances and corrected for chance responding. Recognition functions are based only upon trials on which the detection response was correct.

judgment task and taste substance. Each of the data points has been corrected for chance responding by the familiar formula (Kling \& Riggs, 1971, p. 34),

$$
P_{c}=\frac{P_{o}-P_{g}}{100-P_{g}},
$$

where $P_{c}$ is the corrected percentage of correct responses, $P_{o}$ is the raw observed percentage, and $P_{g}$ is the percentage of correct responses obtainable by guessing alone (50\% in detection and $33 \%$ in recognition). The psychophysical functions for the recognition task are based only upon those trials on which the detection response was correct.

If the odor of a substance contributes significantly to its "flavor," then the T group's performance should be inferior to that of the TS group on that substance. It is also conceivable that for some substances, odor is the more important determinant of discrimination, in which case the performance of the $S$ group should approach or exceed that of the $\mathrm{T}$ group.

The TS group was clearly superior to the $T$ and $S$ groups in the detection of orange juice and chocolate syrup. When vinegar was the substance, the TS group showed scant, if any, superiority to the T group, while the $S$ group performed at chance level on all but the two strongest concentrations.

These data suggest that, within the range of concentrations used, the odors of orange juice and chocolate syrup contributed significantly to the flavors of those substances. The odor of vinegar apparently was not an important component of its flavor at these concentrations. 
One might speculate from the detection data that taste and smell contribute to flavor in additive fashion. However, the recognition data for chocolate suggest that a more complex interactive effect may have been present. On the recognition task, the $\mathrm{T}$ and $\mathrm{S}$ groups performed virtually at the chance level, yet the TS group recognized chocolate nearly as well as they detected chocolate. It is possible that taste and smell function additively in some substances and interactively in others.

One fact that should be considered in interpreting these data is that the $T$ group may not have been discriminating on the basis of "taste" alone. Despite the use of the nose clips, it is possible that some odorous stimuli may have entered the nasal cavity by the nasopharyngeal route. Mozell et al. (1969) eliminated this source of olfactory stimulation by forcing a current of air into the nostrils during tasting. Their "air-flow" subjects showed substantial impairment in the ability to identify common foods compared to TS subjects. Mozell et al. did not, however, include a nose-clip control group. It is not clear whether any appreciable amount of nasopharyngeal olfaction does occur with a nose clip, nor can it necessarily be concluded that the air-flow method is superior to the nose clip in eliminating olfaction.

The identification data in Figure 1 show, as might be expected, that the recognition task was more difficult than the detection task. With the exception of the $S$ group on vineger, all groups attained higher overall percentages of correct responses on detection than on recognition. Except for the TS group on chocolate, all groups did rather poorly on the recognition task. It is possible that the poor recognition performance was due to the recognition task's having a higher absolute threshold than the detection task. The concentrations used were selected to encompass the psychophysical function for the detection task. If the recognition threshold is higher than the detection threshold, it is also possible that a much greater range of concentrations would be needed to generate a complete psychophysical function for the recognition task. There is evidence from a study by Rausch and Serafetinides (1975) that recognition thresholds are substantially higher than detection thresholds. The detection thresholds for pyridine, pentyl acetate, and phenethyl alcohol (expressed in - log molar concentration) were 5.60,7.50, and 9.90, respectively; the recognition thresholds for the same three substances were $3.50,4.20$, and 5.00, respectively. The Rausch and Serafetinides study, incidentally, also reported that subjects with temporal lobectomies had detection thresholds that were elevated to the recognition threshold level. The recognition thresholds were virtually unaffected by the lobectomies.

In general, the recognition data support the conclusions drawn from the detection data, except for the TS group's orange juice results. The TS group was superior to the $\mathrm{T}$ and $\mathrm{S}$ groups in the detection of orange juice, but this superiority was scarcely present in the recognition data. It is possible that since orange juice and vinegar are so similar chemically, the subjects confused the two substances. Such confusion would have no effect on the detection task but would affect recognition.

The data show that on trials when the detection response was correct and the recognition response was incorrect, the TS group was nearly twice as likely to identify orange juice as vinegar than as chocolate $(28 \%$ of total trials vs. $15 \%$ for chocolate). Similar results were obtained with vinegar. TS subjects identified vinegar as orange juice 2.5 times as often as chocolate on detection-correct trials ( $40 \%$ of total trials vs. $16 \%$ ). These differences were not due to response bias, as can be seen on the trials when the detection response was incorrect. Chocolate was chosen more frequently than vinegar on orange juice trials (34\% vs. $18 \%)$ and more frequently than orange juice on vinegar trials (29\% vs. $21 \%$ ).

Practice effects were small, as might be expected with only 18 trials per subject. On the detection task, the uncorrected percentages of correct responses over all groups were $64 \%$ for the first six trials, $72 \%$ for Trials $7-12$, and $72 \%$ for Trials $13-18$. On the recognition task, the percentages were $44 \%, 46 \%$, and $47 \%$, respectively. No significant differences were found as a function of age or sex.

It was concluded that the discrimination of an orally ingested substance may depend upon both the odor and the taste of the substance at near-threshold concentrations. The extent to which odor contributes to discrimination depends upon the intensity of the odor.

\section{REFERENCES}

Brillat-Savarin, J. A. [The physiology of taste: Or meditations on transcendental gastronomy] (M. K. F. Fisher, trans.). New York: Knopf, 1971. (Originally published, 1825.)

Clark, E. C. Anosmic gustation. New England Journal of Medicine, 1968, 278, 337.

Clark, E. C., \& Dodge, H. W. Effect of anosmia on the appreciation of flavor. Neurology, 1955, 5, 671-674. (a)

Clark, E. C., \& Dodge, H. W. Extraolfactory components of flavor. Journal of the American Medical Association, 1955, 159. 1721-1723. (b)

Crosland, H. R., Goodman, M., \& Hockett, A. Anosmia and its effects upon taste perceptions. Journal of Experimental Psychology, 1926, 9, 398-408.

Doty, R. L. Intranasal trigeminal detection of chemical vapors by humans. Physiology \& Behavior, 1975, 14, 855-859.

Gilliland, A. R. The taste sensitivity of an anosmic subject. Journal of Experimental Psychology, 1921, 4, 318-326.

KLING, J. W.,\& RIGGs, L. A. (Eds.). Woodworth and Schlosberg's experimental psychology (3rd ed.). New York: Holt, Rinehart, \& Winston, 1971.

Mozell, M. M., Sмith. B. P., Smith, P. E., Sullivan, R. L., 
\& SWender. P. Nasal chemoreception in flavor identification. Archives of Otolaryngology, 1969, 90, 367-373.

Rausch. R., \& Serafetinides, E. A. Specific alterations of olfactory function in humans with temporal lobe lesions. Nature, 1975, 255, 557-558.

Rubin, E. Taste. British Journal of Psychology, 1936. 27. 74-85.
SChNeIder, R. A. The sense of smell in man. Ne'l England Journal of Medicine, 1967, 277. 303.

(Received for publication February 23, 1979.) 